\title{
Trends in Complex Multiphase Flow Modeling for Effective Fuel Energy Acquisition
}

\author{
Muhammad Talha Tahir ${ }^{1}$, Dengwei Jing1, Muhammad Hatami ${ }^{1,2}$, Hassan Elahi ${ }^{3}$ \\ ${ }^{1}$ International Research Center for Renewable Energy, State Key Laboratory of Multiphase Flow in Power \\ Engineering, Xi' an Jiaotong University \\ Xi' an 710049, China \\ talha1@stu.xjtu.edu.cn; dwjing@xjtu.edu.cn \\ ${ }^{2}$ Mechanical Engineering Department, Esfarayen University of Technology (EUT) \\ Esfarayen, North Khorasan, Iran \\ m.hatami@xjtu.edu.cn \\ ${ }^{3}$ Department of Mechanical and Aerospace Engineering (DIMA), Sapienza University of Rome \\ Rome 00186, Italy \\ hassan.elahi@uniroma1.it
}

\begin{abstract}
Multi-phase reaction engineering and specifically process intensification procedures provide visibly high percentages of lesser energy consumption rate and chemical production costs from both bulk and distributed feedstock. By terminal intensification process, the potential of shale gas for enhancing multiphase reaction engineering technologies also allows significant reduction in the carbon footprint excreted through energy generation fuels and chemical sources. In this research, the flow through various porous domains is investigated as it is of significant concern in many natural and developed zones. This research article enlists a porous medium case study w.r.t to the diameter variations and challenges in multi-phase flow modeling $(\mathrm{mfm})$ for accessing efficient energy resources, analyze latest methods available in the engineering science for acquiring effective and better results by converting energy resources into fuels, chemicals and synthetic concoctions. At the end, this research work concludes with the results of showing enhanced performance rates in porous medium by defining the diameter range over porosity limit and it found to be maximum at 0.4 and -0.6 for 0.3 and 0.8 of porosity limit respectively.
\end{abstract}

Keywords: Multiphase Reaction Engineering, Terminal Intensification Process, Complexity of Multiphase flow modeling, Challenges and Problems, Efficient Energy Resources.

\section{Introduction}

Effects of multiphase flows have been studied broadly for several decades now, as due to their wide applications based in nuclear, petroleum and chemical industries. Lately, the investigational operating's of flow behavior has been greatly amplified by the depth of understanding of the core mechanisms, which resulted in providing the groundwork for enhanced field data for the essential constitutive laws and conservation equations of multiphase flow frameworks in there domains. As the circulating fluidized beds are effectively and extensively used in viable industrial operations now a day, but still much remains to be explored due to the multifaceted nature of the multiphase flow models. And in order to enhance the vital knowledge of complex multiphase flow conduct, much exploration is still needed to be done.

During the recent years computational fluid dynamics in multiphase flow has become an acknowledged and valuable tool in modeling of multiphase flow systems and much development has been already made towards creating computer codes for portraying fluidized beds. According to the available literature, most of the created models depends on a twophase depiction, one phase comprised of gas and one phase for the solids, in which all the units of the domain are presumed to be identical, characterized by diameter, followed by a shape factor, density and restitution coefficient. With utmost all the models using the stage describe by Euler, the constitutive equation of the solid phase is based on the theory of particle flow dynamics based on the work of Jenkins and Savage [1], Lun et al. [2], Ding and Gidaspow [3], and Gidaspow [4], Enwald et al. [5] and Hjertager [6] provides an overview of different Euler two-phase flow models applicable to fluidization. In order to understand the complex multiphase flow system in detail, the experimental work is obviously also important.

In multiphase systems, due to the difference sizes or densities particle segregation will also have a vital play on the flow behavior. And to describe this occurrence, it is necessary to expand multiple particle phases. Jenkins and Mancini [7] propose an extended kinetic theory of granular flow to binary mixtures. A basic assumption is considered for equal 
turbulent flow, with little alteration in temperature of each phase. W. Adamczyk et al. [8] had developed and simulated a model based on this work area using a gas phase and three solid phases. The resulting model predicts the segregation effect very accurately and is very consistent with the experimental data.

Reaction Engineering has also got its impact for converting energy sources into fuels, chemical and synthetic concoctions. If we provide incremental boosts over the old surviving systems and technologies, we cannot attain the required objectives. A current dilemma for energy and synthetic chemicals is the undeniable range of future raw materials and the need to change novelty, while at the same time achieving the goal of expanding energy accessibility through a shrinking natural carbon footprint. [9].

Flow through porous media is important in many natural and developed areas. Examples include the hydrocarbons flow in oil wells, the flow of groundwater through rock formations, and the transport of many minerals and pollutants through the ground. Koo and Kleinstreuer [10] studied the steady laminar flow of nanofluids in microchannels for the conduction- convention heat transfer effects of two different base fluids, water and ethylene glycol with lowconcentration copper oxide nanospheres. Due to the complexity in structure of classic porous media, numerical simulations are generally restricted to procedures using simplified boundary conditions or methods based on conceptual continuous models, so spatially averaged Navier- Stokes equations [11] can be used to consider flow behavior on large (average) scales ; Breugem [12].

Kefayati et al. [13] calculated the non-Newtonian nanofluids in the cavity and entropy production studies were carried out under various conditions. Based on the above brief review of existing literature and work, it can be seen that there is no suitable general reference for the study of porous media on heat transfer effects [15], the generalized multiphase flow model of porous media flow is given here, and the consistency of the model is briefly discussed.

\section{Numerical Methods}

To simulate porous media multiphase flow using physical velocity porous formulations to solve physical velocity fields throughout the flow field, including porous and non-porous domains. Assuming the general scalar of stage ith, $\emptyset_{i}$, the governing equations in isotropic porous media take the following form:

$$
\frac{\partial\left(\gamma \alpha q^{\rho} q \emptyset q\right)}{\partial t}+\nabla \cdot\left(\gamma \alpha q^{p} q \vec{v}_{i} \phi_{i}\right)=\nabla \cdot\left(\gamma \tau_{i} \nabla \emptyset_{i}\right)+\gamma S_{\varnothing, i}
$$

Here $\gamma$ is porosity, which can fluctuate with time and space; $p_{i}$ is phase density; $a_{i}$ is volume fraction; $S_{\varnothing, i}$ is source term; $\vec{v}_{i}$ is phase velocity vector and $\tau_{i}$ is diffusion coefficient. The continuity equation will be as:

$$
\frac{\partial}{\partial t}\left(\gamma \alpha q^{p} q\right)+\nabla \cdot\left(\gamma \alpha q^{p} q \vec{v}_{i}\right)=\gamma \sum_{p=1}^{n}\left(\dot{m}_{p q-} \dot{m}_{q p}\right)+\gamma s_{i}
$$

Energy Equation will be as [14]:

$$
\begin{gathered}
\frac{\partial}{\partial t}\left(\gamma \alpha q^{p} q h_{i}\right)+\nabla \cdot\left(\gamma \alpha q^{p} q \vec{v}_{i} q^{h} q\right)=-\gamma \alpha_{i} \frac{\partial p_{q}}{\partial t}-\gamma \overline{\bar{\tau}} q \\
: \nabla \vec{v} q-\nabla \cdot\left(\gamma \vec{q}_{i}\right)+\gamma s_{i}+\gamma \sum_{p=1}^{n}\left(Q_{p q-} \dot{m}_{p q} h_{p q}-\dot{m}_{q p} h_{q p}\right)+Q_{s p}
\end{gathered}
$$

For porous media with highly anisotropic inertial resistance, enable alternative formulations with inertia resistance. The "Alternative Formulation" option provides better stability for calculations when the porous media is anisotropic. The pressure loss through the media depends on the velocity vector of the ith component in the media. For alternative formulation:

$$
\mathrm{S}_{\mathrm{i}=\frac{1}{2}} \mathrm{pC}_{\mathrm{i}}\left|\mathrm{v}_{\mathrm{i}}\right| \mathrm{v}_{\mathrm{i}}
$$


In a laminar flow through a porous medium, the pressure drop is typically proportional to the velocity, and the constant $\mathrm{C}_{2}$ can be deliberated to be zero. Neglecting convection acceleration and diffusion, the porous media model is then simplified to Darcy's law:

$$
\nabla p=-\frac{u}{a} \vec{v}
$$

The pressure drop in each of the three $(\mathrm{x}, \mathrm{y}, \mathrm{z})$ coordinate directions in the porous region calculated by Ansys fluent is:

$$
\begin{aligned}
& \Delta p_{x}=\sum_{j=1}^{3} \frac{\mu}{\alpha_{x j}} v_{j} \Delta n_{x} \\
& \Delta p_{y}=\sum_{j=1}^{3} \frac{\mu}{\alpha_{y j}} v_{j} \Delta n_{y} \\
& \Delta p_{z}=\sum_{j=1}^{3} \frac{\mu}{\alpha_{z j}} v_{j} \Delta n_{z}
\end{aligned}
$$

Where $1 / a_{i j}$ is an entry in matrix $\mathrm{D}$, vj is the velocity component in the $\mathrm{x}, \mathrm{y}$, and $\mathrm{z}$ directions and $\Delta n_{x}, \Delta n_{y}$, and $\Delta n_{z}$, is the thicknesses of the medium in the $\mathrm{x}, \mathrm{y}$, and $\mathrm{z}$ directions. The exact $\mathrm{k}-\varepsilon$ equations contain many unknown and immeasurable terms. For more practical methods, use the standard k- $\varepsilon$ turbulence model (William G in 1976 [23]) which is based on our knowledge of the relevant processes, thereby minimizing the unknowns and presenting a set that can be applied to a large amount of turbulence applications. For turbulent flow energy k; the k epsilon ( 2 equations) (realizable) for standard wall functions is given as, and the boundary conditions will be $\mathrm{C}_{2}$-Epsilon at 1.9 values; TKE Prandtl no. at 1; TDR Prandtl no. at 1.2.

$$
\frac{\partial(p k)}{\partial}+\frac{\partial\left(p k u_{i}\right)}{\partial x_{i}}=\frac{\partial}{\partial x_{i}}\left[\frac{u_{t}}{\sigma_{k}} \frac{\partial k}{\partial x_{j}}\right]+2 \mu_{t} E_{i j} E_{i j}-\rho \epsilon
$$

\section{Challenges}

We feel major reaction engineering concerns and challenges in refining domain of fuels, where combined shale gas and oil, including heavy oil, has led to a bifurcation in raw material, with necessity for low-intensity paraffin upgrading and heavy oil conversion methods and techniques [17]. As with the details about multiphase measuring techniques and how they can be implemented in the research areas, there are a lot of problems and missing elements in the calculations hence concluded. Therefore, by closely analyzing some situations [18] I have proposed some solutions for specific challenges in different scenarios.

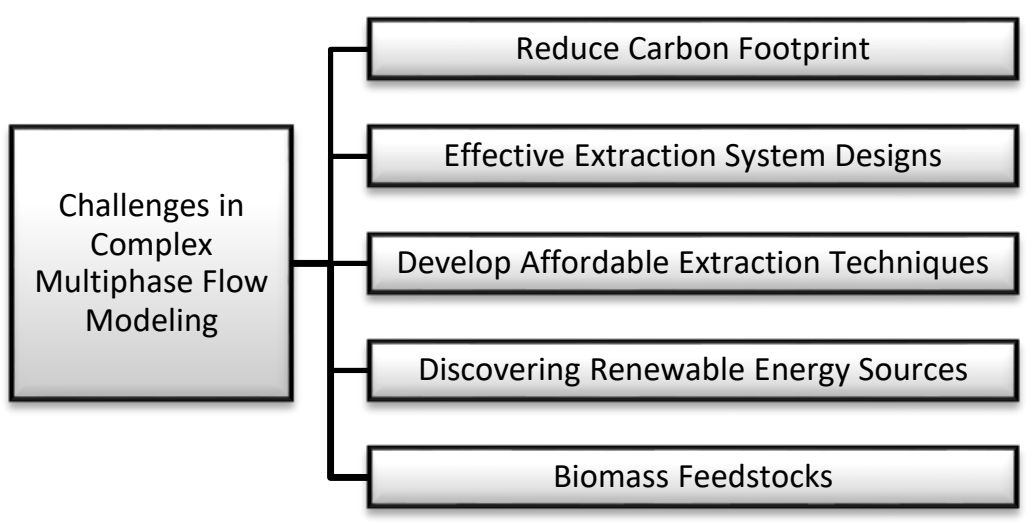

Fig. 1: Challenges in Complex Multiphase Flow Modeling.

\subsection{Reduce Carbon Footprint}

Ample gas disposal offers new enticements to transform methane gas into chemical building blocks for example propylene gas, ethylene gas and aromatic hydrocarbons fluids, it is also converted to liquid-based gasoline chemicals and diesel fuel. So some latest reactor designs are highly required to enhance and lower the carbon concentration during the conversion processes. A section of available gas is present in a smaller sized storage container or in an area including an offshore geographical location. 


\subsection{Effective Extraction System Designs}

As for power generation at lowered and much compact carbon footprint methane itself is easy to burn, and for its exchange to liquids and chemical fuels for example diesel, gasoline, or methanol and few of its derivative chemical compositions do indeed require intense high temperature endothermic (reductive based) or exothermic (oxidative based) coupling reactions, where differentiated conversion and thermal-heat transfers are based on serious design issues.

\subsection{Develop Affordable Extraction Techniques}

The maturity of the less expensive modular extraction systems used to disperse the production of fuels and chemicals to reduce the need to burn residues, which is the goal of effectively producing these resources. Heavy oil is now another resource for upgrading and transformation, requiring multiphase reactor development techniques. Therefore, this raw material is important to resource protectors because the major part of the reserve is known. Energy pricing for fuel extraction and conversion still requires the development of efficient processes that must be supplemented by favorable carbon capture, which also requires the development of solutions for multiphase reaction engineering.

\subsection{Discovering Renewable Energy Sources}

As a result of integrated energy, demand and sustainability drivers, a wider range of raw materials and resource choices are needed, from fossil to renewable, using more skilled and enhanced designs. Therefore, we can suggest that energy conversion is also increasing stress on renewable energy, the reaction engineering challenges in wind and solar storage, and the use of captured carbon dioxide (carbon dioxide) or other energy carriers to convert into molecular fuels.

\subsection{Biomass Feedstocks}

The use of the latest biomass feed stocks presents multiple challenges in conversion technology. We have seen that bio-based feedstock is naturally moist, and the distinction between fuel or chemical products and water molecules is often energy-intensive. Due to the low energy density of raw materials relative to fossil hydrocarbons, the largest bio-refinery conversion facilities are limited to a certain limit by supply logistics. Therefore, we can say that this means extremely intensive processes and manufacturing facilities to provide economics that can compete with large refineries.

\section{Porous Domains - Casestudy}

Porous fluid problems come across in lots of engineering applications along with industrial and manufacturing scales, in soils, in food, in filters, etc. Many engineering applications, such as filters, catalyst beds and packaging, involves the flow modeling through porous media. This case study specifically solves a problem involving gas flow through porous domains.

\subsection{Addressed Statement}

Nitrogen flows through the inlet at a uniform velocity of $22.4 \mathrm{~m} / \mathrm{s}$, passes through a ceramic monolithic substrate with square channels, and then exits through the outlet while the absorber tube is filled with porous media.

The case validates how to create a porous domain region for the substrate with appropriate resistances; to calculate results for gas flow in porous area using the pressure solver, and using the X-Y plots to determine the degree of non-uniformity of the pressure drop across the substrate and the flow through the cross-section of the geometry.

As per some meshing details, the soothing would be medium; the minimum edge length is $125.6 \mathrm{~mm}$, joined by the Boolean unity. The system is conventional to time steady, the model to viscous $\mathrm{K}$ epsilon 2 equations, fluid is nitrogen with a density $\left(\mathrm{kg} / \mathrm{m}_{3}\right)$ of 1.138 and viscosity $(\mathrm{kg} / \mathrm{m}-\mathrm{s}) 1.663 \mathrm{e}-05$, as the porous zone is enabled the flow is laminar, the values for viscous resistance and inertial resistance at direction- 2 and direction- 3 are set to any large numbers, which are many orders of magnitudes larger than the direction-1 flow values are numerous orders of scale greater than that of Direction-1 flow and will not mark any radial flow, velocity at inlet is set at $22.4 \mathrm{~m} / \mathrm{s}$, turbulent Intensity value at $10 \%$ for inlet, Gauge Pressure

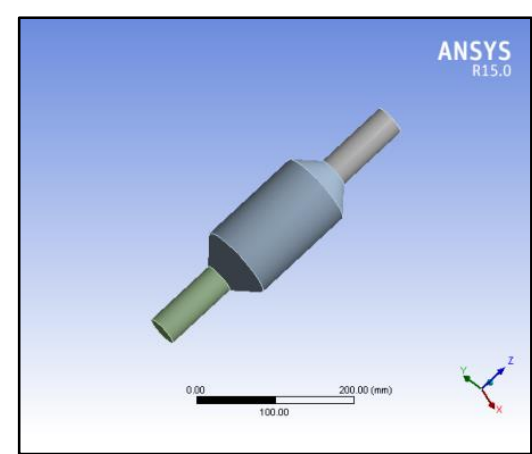

Fig. 2: Model for the Porous Medium Geometry.

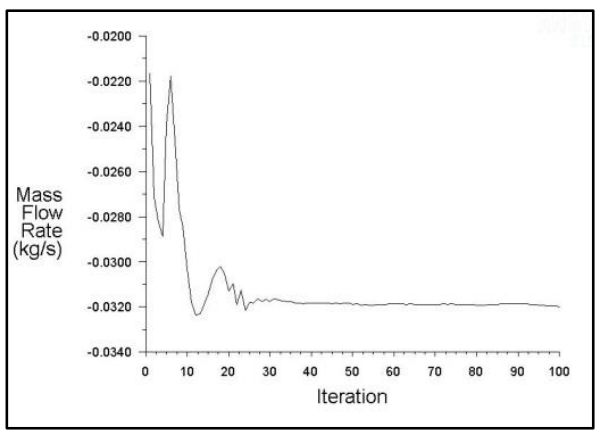

Fig. 3: Surface Monitoring of Mass flow Rate.

0 at the outlet and with turbulent intensity value at $5 \%$. The turbulent dissipation rate $\left(\mathrm{m}_{2} / \mathrm{s}_{3}\right)$ will be 1185.214. Converging calculation for mass flow rate flattens out at about 100 iterations as displayed in figure 3. 


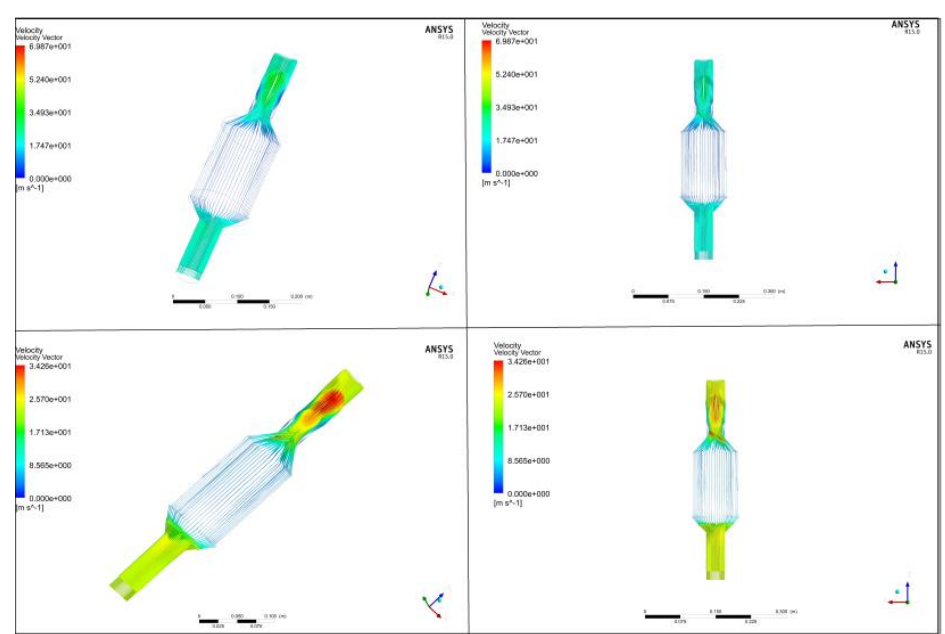

Fig. 4: Velocity Vector Profiles along local and global range.

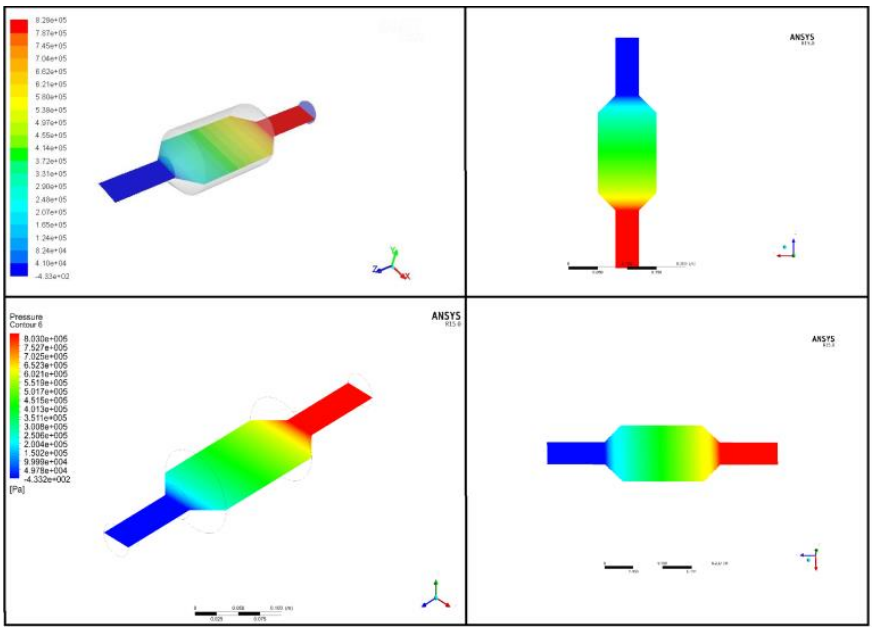

Fig. 5: Pressure Contours on Plane 4 (Median Cut).

The flow direction and plots shown in Figure 4. suggests that the flow enters as a jet with recirculation on both sides. As it passes through the porous region, it decelerates and straightens and exhibits a more uniform velocity profile. This makes the metal catalyst present in the substrate more efficient. The velocity at the center before the nitrogen enters the substrate is very high and then decreases as it passes through and exits the substrate. The green area corresponds to a moderate velocity plot and its range increases.

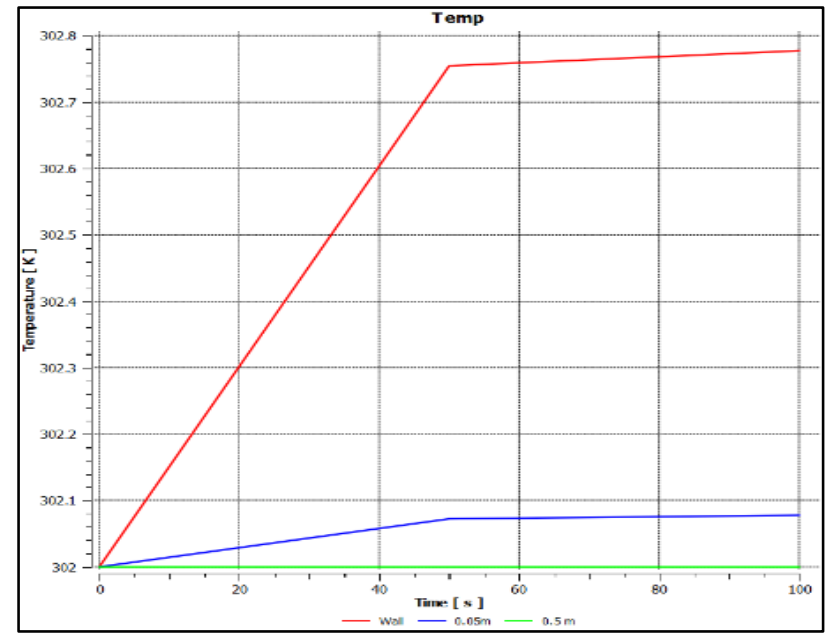

Fig. 6: Temperature Profiles along axis and Planes at $0.05 \mathrm{~m}$ and $0.5 \mathrm{~m}$.

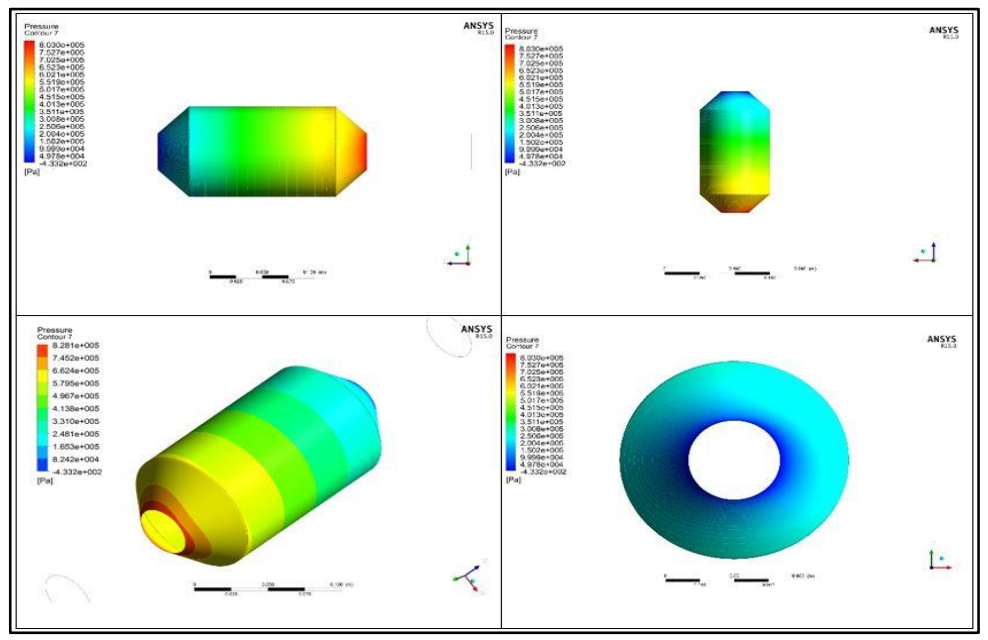

Fig. 7: Pressure Contours at Porous Domain.

The pressure contour shown in figure 5 change rapidly in the middle portion, where the fluid velocity change as it pass through the porous region. The pressure drop can be high due to the inertia and viscous resistance of the porous media. Defining and to determine this pressure drop are one of the aims of CFD analysis.

Flow non-uniformities are quickly detected by images of velocity vectors and pressure profiles. Surface integration and xy plots provide pure digital data in the figure 5,6 and 7. The temperature profiles at different planes and on the wall, the behavior can be examined by figure 6 . You can get a more precise solution by using the suitable high-order discretization pattern and adjusting the grid. Grid adaptation also certifies that the solution is independent of the mesh.

The extent amid the average, maximum and the minimum value of the $X$ velocity gives an indication of the uneven velocity distribution. And for the value of mass-weighted average for the X velocity at $y-130$ and $y-95$ the net would be 3.6264589 . 
The accuracy of grid independence testing and computational verification is based on ANSYS Fluent, which solves the problem of three-dimensional conjugate heat transfer in the porous domain. To identify minimum mesh dependencies, consider a structured grid of four mesh numbers for grid independence testing. Figure 8 shows the results of mass flow rate calculated on different meshing size and number of elements. As the calculated relative differences in mass flow rate variations are less than $0.1 \%$ over the mesh elements range of 2.5 to 5 million, respectively, so 3.5 million cells are considered suitable for all computational simulations performed in this study.

\section{Trends}

\subsection{Porous Media Patterns}

By following the trends at different diameters and by plotting the results in an appropriate way we hence conclude the results shown in the figure 9. By changing the diameter of the substrate region we have different effects on the results. The velocity and pressure drops depend upon the chosen diameter for the duct.

Now by simulating the results across different radial positions we get a variety of data and which is shown according to their peak points respectively, in figure 10 . With the increase in diameter the trends are shown in the figure.

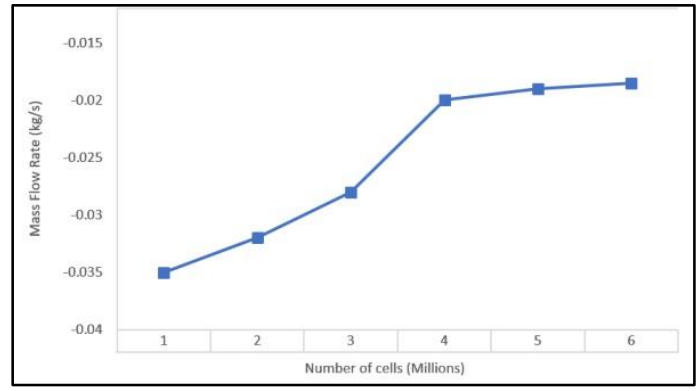

Fig. 8: Grid Independence Testing for the ceramic monolith porous substrate.

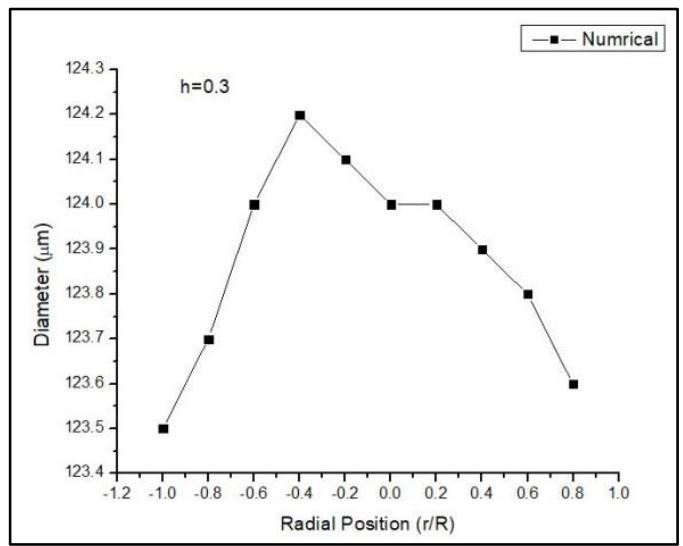

Fig. 9: Diameter Trends.

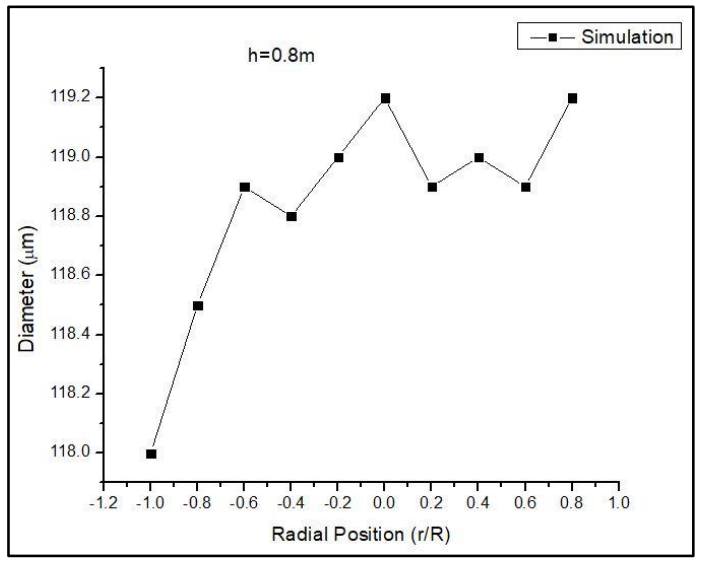

Fig. 10: Diameter variations w.r.t radial positions.

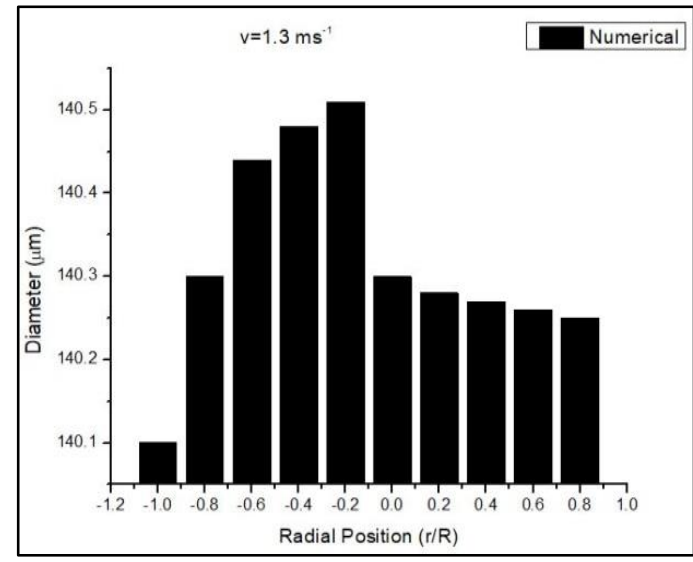

Fig. 11: Diameter variations via numerical simulations.

In order to solve the above problem the numerical solution to the point is at $\mathrm{v}=1.3 \mathrm{~ms}^{-1}$. As the diameter is changed to the extent the heat transfer through the porous domain increases but it is up to one limit and after the point at 124.2 we get the divergence of peak in the opposite direction. From Fig. 9, it is evident that the trend of radial position vs. diameter increases linearly up to radial position of -0.4 and is maximum at this point. After this point the trend decreases for $h$ at 0.3m. In Fig. 10, the simulation is performed at the same conditions as performed in Fig. 9 but at $0.8 \mathrm{~m}$ of h. The Fig. 10 represents the nonlinear behavior of radial position over diameter, it is found to be maximum at 0 of radial position. Moreover, the numerical simulation is performed to predict the diameter against the radial position for velocity of 1.3 $\mathrm{ms}^{-1}$ as represented in Fig. 11. It is observed that at this particular velocity the diameter is found to be maximum at -0.2 of the radial position and found to be minimum at -1 of radial position.

\subsection{Current Research Domains}

By observing, the most cited various research articles about multi-phase flow modeling, on Elsevier journals and other research repositories over the internet, three major categories of research are focused for this paper, i.e. survey 
papers, experimental papers and visualization modeling papers. A visual representation of these categories according to each year is given in figure 12 .

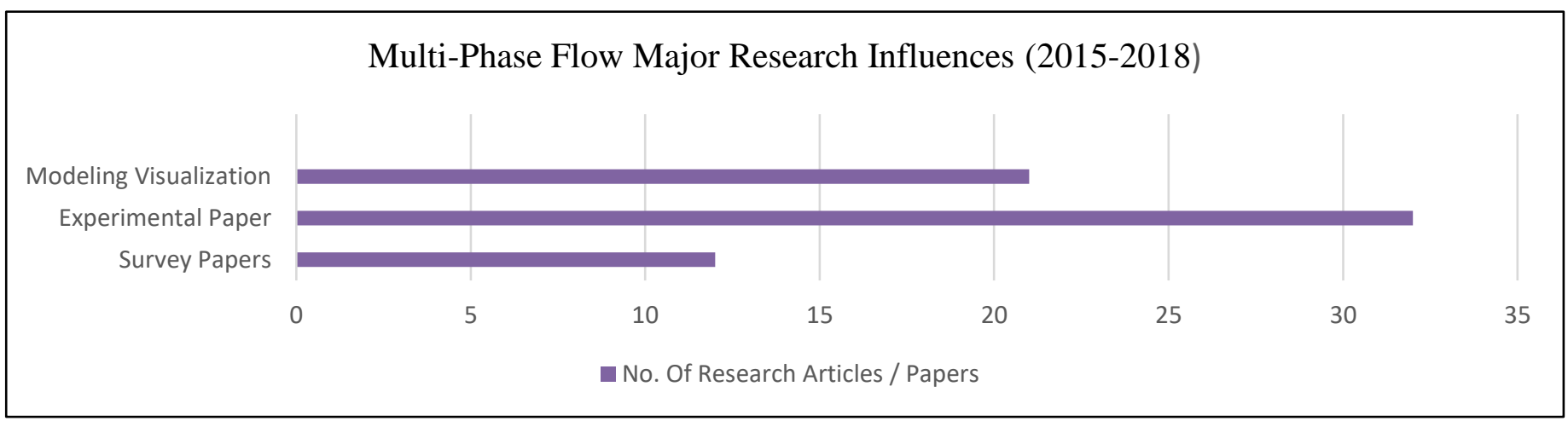

Fig. 12: Multi-Phase Flow Major Research Influences (2015-2018).

\subsection{Latest Industry Practices}

As we know the use of simulation based software, have increase in all industries including the multi-phase flow modeling to conduct experiments and researches [16]. For software forums and platforms regarding visual modeling and simulation of commercial and experimental systems, many platforms are used. In modeling, the performance matrix must be validated via proved experiments to be considered authentic, often using the multi-phase performance throughout reactors [19]. For this purpose, a collection of many software and simulations are being utilized, which provide a predictive capability which is indeed a saving time tool and also provides cost estimations in preparation [20]. For this research, much such software including their usage, rating and users review have been analyzed and a graph showing the most widely used [20] along with their usage percentage is shown in the figure 13.

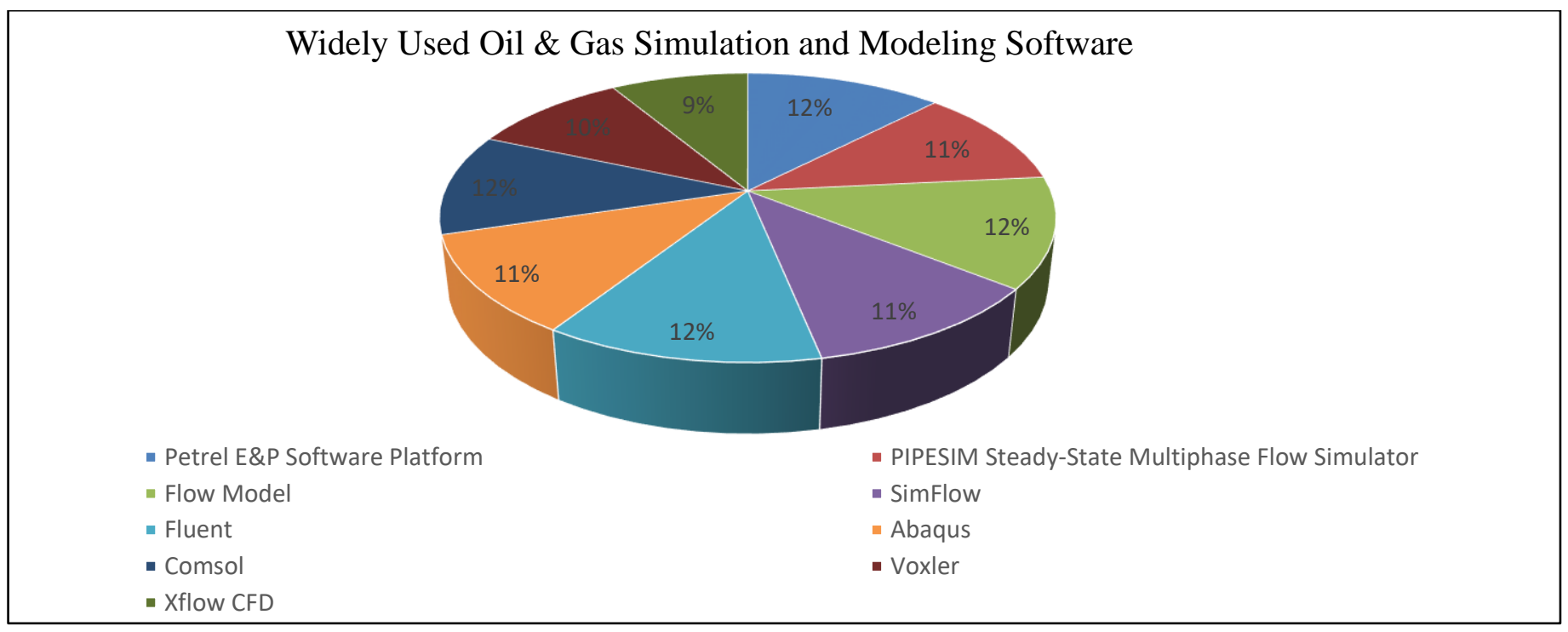

Fig. 13: Widely Used Oil \& Gas Simulation and Modeling Software.

\section{Conclusion}

\subsection{Remarks}

The oil and gas industry statistics of more than twenty years show that MFM is a stable field of research. Liquefied natural gas (LNG) has the potential to be used as modular substitute for electrical power generating utility gas. In fuel markets, gas-to-liquids (GTL) is one of the growing products also. Our main focus is that the great availability of natural fuels like gas makes GTL a very feasible option, but process intensification is needed for purifying the fuel as well as to reduce capital costs [14]. The results acquired hence require design and development of intensified multi-phase reactors including all three types of matter, solid, liquid and gas. 
The intensification process has vitally decreased the energy consumption and production cost of chemicals and fuel. In the past decade, major industrial and research advancements have been conducted which clearly states the potential of this process in multi-phase flow modeling. Although process intensification in the modeling and design of complex systems presents challenges for cohesive heat and mass transfer steps, these steps are also superimposed on chemical reactions [21]. Despite there is not standardized generic multiphase flow measurement solution for specific applications, still the desired results can be obtained by enhancing pre-development operation parameters. The MFM has 2D and 3D [22] micro level structure development, containing radiation -induced segregation, tributary phase nucleation, cavity migration, cavity and gas bubble super lattice format ion, grain growth and hydrate formation.

Few of the procedures deploy standardized methods applied effectively. While, the other methods opt for single phase flow measuring techniques for dual phase flows. Altogether, these methods increase the understanding of the underlying concept of dual-phase flows and enhance the performance rates in these techniques.

\subsection{Summary}

Rapidly changing climatic patterns required the significant need of reduction in carbon footprint; for this, shale gas revolution has potential to provide more means of energy production with less negative impact on. In this target, challenges vary from high to low-level categories. Development process of chemical manufacturing in multi-scale investigation and multi-phase flow modeling provides tools and techniques for better, faster and cost-effective units.

In addition, process intensification is one of the possible solutions to overcome these challenges and to address them in a more appropriate way. And to eradicate the adverse effects of new energy systems and extraction methods of shale gas the multiphase flow modeling with process intensification as in addition is vital.

As through the multiphase flow models in porous mediums we keep to know this fact that heat transfer is indeed efficient through the porous domains when selecting the suitable diameter range. In this research, enhanced performance rates in porous medium by defining the diameter range over porosity limit is investigated and it found to be maximum at -0.4 and -0.6 for 0.3 and 0.8 of porosity limit respectively. Moreover, the diameter is found to be maximum at -0.2 of the radial position and found to be minimum at -1 of radial position.

To meet viable production needs and survive in a global competitive market, integrated process intensification provides a favorable opportunity for the rigorous process of business development. For optimal process configurations, process system engineering helps develop new process enhancement technologies by providing systematic regulations.

\section{References}

[1] J. Jenkins and S. Savage, "A theory for the rapid flow of identical, smooth, nearly elastic, spherical particles," Journal of Fluid Mechanics, vol. 130, no. 1, pp. 187, 1983.

[2] C. Lun, S. Savage, D. Jeffrey and N. Chepurniy, "Kinetic theories for granular flow: inelastic particles in Couette flow and slightly inelastic particles in a general flowfield," Journal of Fluid Mechanics, vol. 140, no. 1, pp. 223, 1984.

[3] J. Ding and D. Gidaspow, "A bubbling fluidization model using kinetic theory of granular flow," AIChE Journal, vol. 36, no. 4, pp. 523-538, 1990.

[4] Gidaspow, Multiphase Flow and Fluidization. Boston: Academic Press, 1994.

[5] H. Enwald, "Eulerian two-phase flow theory applied to fluidization," International Journal of Multiphase Flow, vol. 22, pp. 21-66, 1996.

[6] B. H. Hjertager, "Computational fluid dynamics (CFD) analysis of multiphase chemical reactors," Delft University of Technology, 1997. Also trends in Chem. Eng. 4, pp. 45-92, 1998.

[7] J. Jenkins and F. Mancini, "Balance Laws and Constitutive Relations for Plane Flows of a Dense, Binary Mixture of Smooth, Nearly Elastic, Circular Disks," Journal of Applied Mechanics, vol. 54, no. 1, pp. 27, 1987.

[8] W. Adamczyk, A. Klimanek, R. Białecki, G. Węcel, P. Kozołub and T. Czakiert, "Comparison of the standard Euler-Euler and hybrid Euler-Lagrange approaches for modeling particle transport in a pilot-scale circulating fluidized bed," Particuology, vol. 15, pp. 129-137, 2014.

[9] Shell Scenarios. (2018, Dec 06). [Online]. Available: http://www.shell.com/global/future-energy/scenarios.html.

[10] J. Koo and C. Kleinstreuer, "Laminar nanofluid flow in microheat-sinks," International Journal of Heat and Mass Transfer, vol. 48, no. 13, pp. 2652-2661, 2005.

[11] S. Whitaker, The Method of Volume Averaging. Dordrecht: Kluwer Academic Publishers, 1999.

[12] W. P. Breugem, "The influence of wall permeability on laminar and turbulent flows," Ph.D. dissertation, Delft University of Technology, Delft, The Netherlands, 2004. 
[13] G. Kefayati, S. Hosseinizadeh, M. Gorji and H. Sajjadi, "Lattice Boltzmann simulation of natural convection in tall enclosures using water/SiO2 nanofluid," International Communications in Heat and Mass Transfer, vol. 38, no. 6, pp. 798-805, 2011.

[14] D. Wood, C. Nwaoha and B. Towler, "Gas-to-liquids (GTL): A review of an industry offering several routes for monetizing natural gas," Journal of Natural Gas Science and Engineering, vol. 9, pp. 196-208, 2012.

[15] N. Moelans, B. Blanpain and P. Wollants, "An introduction to phase-field modeling of microstructure evolution," Calphad, vol. 32, no. 2, pp. 268-294, 2008.

[16] S. Dong and J. Shen, "A time-stepping scheme involving constant coefficient matrices for phase-field simulations of two-phase incompressible flows with large density ratios," Journal of Computational Physics, vol. 231, no. 17, pp. 5788-5804, 2012.

[17] Schwarzkopf, John D., et al, Multiphase flows with droplets and particles. CRC press, 2011.

[18] L. Ruspini, C. Marcel and A. Clausse, "Two-phase flow instabilities: A review," International Journal of Heat and Mass Transfer, vol. 71, pp. 521-548, 2014.

[19] M. Arshadi, M. Piri and M. Sayed, "Proppant-packed fractures in shale gas reservoirs: An in-situ investigation of deformation, wettability, and multiphase flow effects," Journal of Natural Gas Science and Engineering, vol. 59, pp. 387-405, 2018.

[20] W. Song, J. Yao, J. Ma, H. Sun, Y. Li, Y. Yang and L. Zhang, "Numerical Simulation of Multiphase Flow in Nanoporous Organic Matter With Application to Coal and Gas Shale Systems," Water Resources Research, vol. 54, no. 2, pp. 1077-1092, 2018.

[21] X. Shi, S. Finsterle, K. Zhang and D. Lu, "Advances in Multiphase Flow and Transport in the Subsurface Environment," Geofluids, pp. 1-2, 2018.

[22] L. Pan, C. Oldenburg, B. Freifeld and P. Jordan, "Modeling the Aliso Canyon underground gas storage well blowout and kill operations using the coupled well-reservoir simulator T2Well," Journal of Petroleum Science and Engineering, vol. 161, pp. 158-174, 2018.

[23] William G. Gray and Kevin O’Neill, "On the general equations for flow in porous media and their reduction to Darcy's Law," Water Resources Research, vol. 12, no. 2, pp. 148-154, 1976. 